\title{
Capacitación Docente para la Atención a la Diversidad en Futuros Docentes de Educación Secundaria
}

UT. Revista de Ciències de l'Educació

Juny 2014. Pag. 83-96

ISSN 1135-1438

http://pedagogia.fcep.urv.cat/revistaut

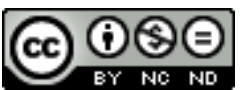

\author{
Ma Jesús Colmenero Ruiz $^{a}$ y $\mathbf{M}^{\mathbf{a}}$ del Carmen Pegalajar Palomino ${ }^{\mathrm{b}}$ \\ Rebut: 05/03/2014 Acceptat: 29/05/2014
}

\begin{abstract}
Resumen
Este trabajo pretende examinar las percepciones de futuros docentes de Educación Secundaria acerca de la formación inicial recibida sobre atención a la diversidad. Para ello, se ha utilizado una metodología descriptiva, llevándose a cabo la recogida de datos mediante el cuestionario tipo Likert elaborado para tal fin $(n=99)$. Los resultados obtenidos demuestran cómo el alumnado considera que el Máster les ha permitido aumentar su interés hacia el estudio de la diversidad y afianzar su elección profesional hacia la docencia; este título les ha permitido adquirir suficientes conocimientos sobre la identificación de las necesidades educativas en el alumnado y los recursos y servicios prestados desde el sistema educativo, entre otros. No obstante, se aprecian diferencias estadísticamente significativas en las valoraciones del alumnado del Máster según la especialidad por la que éstos han accedido a estos estudios de posgrado.
\end{abstract}

Palabras clave: atención a la diversidad, formación docente, Educación Secundaria, Máster.

\section{Teacher Training for Attention to Diversity in Future Teachers of Secondary Education}

\section{Abstract}

This paper aims to examine the perceptions of secondary school student teachers about their initial training on attention to diversity. To do this, we used a descriptive methodology, carrying out data collection through the Likert questionnaire

\footnotetext{
${ }^{a}$ Departamento de Pedagogía. Universidad de Jaén - Jaén

${ }^{b}$ Departamento de Educación. Universidad Católica de Murcia - Murcia
} 
developed for this purpose $(n=99)$. The results show how the students think that the Master has allowed them to increase their interest in the study of diversity and enhance their career choice to teaching; This title has enabled them to acquire sufficient knowledge about the identification of educational needs pupils and the resources and services from the education system, among others. However, statistically significant differences in the evaluations of the students of the Master appreciated by specialty for which they have agreed to these graduate studies.

Keywords: attention to diversity, teacher education, secondary education, Master.

\section{Introducción}

Inmersos en el proceso de convergencia europea en Educación Superior, la formación inicial del docente para la etapa de Educación Secundaria Obligatoria se ha organizado en torno al desarrollo de un Máster Universitario en Profesorado de Educación Secundaria Obligatoria y Bachillerato, Formación Profesional y Enseñanza de Idiomas. La necesidad de esta nueva titulación responde a los serios problemas detectados en la educación a partir de investigaciones, resultados de evaluaciones internacionales o informes de comisiones de expertos (Rocard et al., 2007); por ello, este Máster ha sido concebido y planteado para contribuir a esta necesaria renovación de la enseñanza y no sólo para incorporar nuevos profesores al sistema educativo (Vílchez y Gil, 2007).

El desarrollo de un programa formativo de esta naturaleza debe ofrecer a los futuros profesores unos conocimientos psicopedagógicos y didácticos más profundos, a la vez que ampliar el período de formación de prácticas docentes en centros de secundaria. Además, y coincidiendo con Buendía et al. (2011), la finalidad de esta titulación no es sólo la de capacitar a profesores que resuelvan situaciones educativas sino también, profesionales formados en la reflexión, resolución de problemas, investigación e innovación, que puedan contribuir a que las futuras generaciones de alumnos estén mejor preparadas para afrontar los retos que se les presenten.

El Informe McKinsey (2007) especifica que la calidad de un sistema educativo nunca estará por encima de la calidad de sus docentes, de ahí que sea prioritario que esforzarse en elaborar planes y programas educativos de calidad. De igual modo, diversas investigaciones (Clotfelter, Ladd y Vidgor, 2007; Gustafsson, 2003; Scheneider y Stern, 2010) ponen de manifiesto la relación que se establece entre la formación del profesorado, sus prácticas y el nivel de logro que alcanza el alumnado. Desde esta perspectiva, resulta prioritario analizar la formación pedagógica de los docentes de Educación Secundario para, de este modo, mejorar las prácticas docentes inclusivas en los centros educativos $y$, con ello, el proceso de enseñanza y aprendizaje del alumnado con necesidades específicas de apoyo educativo. 
La finalidad del Máster Universitario en Formación del Profesorado de Educación Secundaria Obligatoria y Bachillerato, Formación Profesional y Enseñanza de Idiomas, es la de proporcionar al profesorado la formación pedagógica y didáctica necesaria y obligatoria para el ejercicio de la profesión docente con arreglo a lo establecido en los objetivos y competencias reflejados en la Ley Orgánica 2/2006 de 3 de Mayo de Educación y la Orden ECI/3858/2007 de 27 de diciembre por la que se establecen los requisitos para la verificación del Máster. En la planificación de las enseñanzas del Título se ha seguido y respetado la propuesta de Módulos de la Orden $\mathrm{ECI} / 3858 / 2007$, en la que se determina un módulo de materias de carácter genérico (Aprendizaje y desarrollo de la personalidad, Procesos y contextos educativos y Sociedad, familia y educación, con un total de 12 créditos), un módulo de carácter especializado (Innovación Docente e Investigación Educativa, Complementos de Formación Disciplinar en la especialidad correspondiente y Aprendizaje y Enseñanza de las Materias de la especialidad, con un total de 24 créditos) y un tercer y último bloque en el que el alumno habrá de desarrollar prácticas docentes en centros de Secundaria (10 créditos) y por último, un Trabajo Fin de Máster (6 créditos). Por ello, una de nuestras preocupaciones como profesoras de la materia de Procesos y Contextos Educativos (4 créditos) del módulo genérico del Máster de Secundaria Obligatoria y Bachillerato, Formación Profesional y Enseñanza de Idiomas, es la de conocer y analizar las percepciones y actitudes de los profesionales que se forman en este Máster en torno a la atención a la diversidad.

En este sentido, el trabajo desarrollado por Sánchez (2007) revela cómo para el desarrollo de una educación de calidad para todos, no existe una adecuada formación inicial del docente, e incluso se parte de prejuicios, creencias e ideas que lo dificultan. Ello viene a reforzar la idea de Pérez (2010) para quién las instituciones formativas se encuentran lejos del ideal que supone la formación de docentes competentes, dada la necesidad de reinventar la profesión para conseguir una educación para todos (Forteza, 2011). Por su parte, la investigación desarrollada por Sánchez y Boix (2008) expone cómo los futuros docentes de Educación Secundaria consideran que la heterogeneidad educativa y la diversificación pedagógica han de estar presentes en la formación inicial del profesorado de Secundaria; no obstante, admiten tener un desconocimiento absoluto acerca del concepto de atención a la diversidad, factores relacionados con la heterogeneidad educativa, necesidades educativas especiales, estrategias y recursos de integración e inclusión en centros y aulas ordinarias, competencias metodológicas, personales y participativas necesarias para transformar el aula y los entornos de la comunidad de aprendizaje en espacios abiertos al aprendizaje desde el diálogo.

Tomando como referencia al profesorado de Educación Secundaria en ejercicio, Colmenero (2007) realiza una investigación cuyo objetivo es indagar las concepciones y percepciones así como las necesidades formativas que éstos demandan sobre los procesos de atención a la diversidad. De forma progresiva, se observa cómo el profesorado se encuentra cada vez más concienciado de la importancia de mantener una actitud positiva hacia la diversidad. De todas formas, y según los datos, se 
constata que la teoría y la filosofía de la integración si la aceptan mayoritariamente pero, a nivel particular, tienen más dificultades para llevar a cabo este proceso de atención a la diversidad. En relación a las necesidades formativas, cada vez se reafirma más la postura de que al profesorado lo que le interesa más son cuestiones de tipo práctico y dinámico, demandando el desarrollo de actividades formativas vinculadas con las distintas formas de planificar actividades y tareas para el alumnado con necesidades educativas especiales, así como conocimientos acerca de cómo evaluar a éstos. A nivel organizativo, lo que más demanda el profesorado es formación acerca de estructuras de apoyo con las que ofrecer una respuesta educativa de calidad al alumnado.

Más recientemente, Alegre (2013) exponen cómo los estudiantes del Máster de Formación del Profesorado de Educación Secundaria de la Facultad de Educación de la Universidad de La Laguna manifiestan actitudes que se relacionan con el deseo de eliminar barreras hacia la inclusión y la preocupación por tener la mejor formación para responder a las peculiaridades del alumnado con necesidades de apoyo educativo. Dichas actitudes han de forjarse desde los primeros momentos de la formación de los futuros maestros teniendo en cuenta que las mujeres suelen presentar actitudes más favorables hacia la inclusión y que la edad de 28 a 32 años se manifiesta crítica a la hora de considerar las actitudes presentes en las dimensiones analizadas en este estudio. En cuanto al tipo de barreras conceptuales, declarativas y estratégicas para desarrollar competencias inclusivas e interculturales podemos decir que son barreras vinculadas con la necesaria colaboración y relación con las familias y con el hecho de lograr unos centros verdaderamente inclusivos; para ello, la formación y preparación del profesorado se manifiesta como el elemento más destacado. Como consecuencia, Pestano y Alegre (2013) llegaron a la conclusión de que existe predisposición por parte del alumnado del Máster de fomentar actitudes hacia la inclusión e interculturalidad motivados, principalmente, por la necesidad de que todo el mundo se sienta acogido (culturas Inclusivas), apostando en coordinar todas las formas de apoyo para atender la diversidad de los centros (políticas Inclusivas) e incentivando una filosofía, creencias y valores que defiendan una educación que requiere del aprendizaje de todos: colaboración, participación, cooperación, etc. (prácticas inclusivas). Del análisis de las actitudes y percepciones de las prácticas inclusivas, se deduce que el mayor porcentaje de la muestra encuestada considera importante y necesario trabajar unidades didácticas dirigidas a la atención del alumnado y la equidad en los recursos para satisfacer sus necesidades. No obstante, es preciso seguir trabajando para el desarrollo de tareas vinculadas a la inclusión educativa en la formación del profesorado de Educación Secundaria sobre todo en lo referente a las relaciones familia-escuela.

Además, Arranz, García y Liesa (2013) se implicaron en una investigación cuyo propósito era analizar si el pensamiento del futuro profesor es más inclusivo tras cursar la asignatura denominada "Atención a los alumnos con necesidad específica de apoyo educativo" (asignatura optativa ofertada en el Máster en Formación del Profesorado de Educación Secundaria Obligatoria, Bachillerato, Formación 
Profesional y Enseñanza de Idiomas en la Universidad de Zaragoza). La conclusión más clara es que el pensamiento de estos futuros profesores es más inclusivo tras cursar esta asignatura optativa, es decir, se evidencia que una mayor formación respecto a las necesidades específicas de apoyo educativo tiene una repercusión en sus creencias y actitudes, haciéndolas más integradoras.

En esta misma línea, Pegalajar, Colmenero y Pérez (2013) concluyeron en una investigación cómo los futuros docentes de Educación Secundaria son conscientes de sus carencias formativas poniendo, a la vez, de manifiesto la necesidad de incorporar dichos aspectos en los planes de estudio de esta titulación inmersa ya en el proceso de convergencia europea. En este sentido, autores como Calvo y Susinos (2010) y Vigo, Soriano y Julve (2010) establecen las directrices de lo que debería incluir un programa de formación inicial del profesorado en el ámbito de la Educación Especial, siendo éste: visión clara de la enseñanza a impartir, currículum fundamentado en la práctica, experiencias clínicas, estándares de la práctica, relaciones entre la Universidad y la escuela, empleo de estudios de caso, indagación y evaluación y análisis reflexivo y crítico (Santos, 2010). Así pues, el docente podrá ofrecer ayudas pedagógicas adecuadas a todo el alumnado una vez cuente con elementos teóricos y prácticos que permitan favorecer el aprendizaje (Sykes, Bird y Kennedy, 2010; Conklin, 2012).

Finalmente, López, Echeita y Martin (2009) construyeron un cuestionario de dilemas que permitió caracterizar las concepciones de profesores y orientadores acerca del proceso de inclusión educativa, centrándose en la discapacidad intelectual en la Enseñanza Secundaria. Plantean un esquema conceptual de doce subdimensiones que representan núcleos de contenido relevantes para abordar las distintas perspectivas acerca de los procesos de inclusión, organizadas en tres dimensiones: teorías sobre las dificultades de aprendizaje, perspectivas sobre la organización escolar y valores educativos. El cuestionario fue aplicado a una muestra conformada por 51 profesionales en ejercicio (profesores de Secundaria y orientadores) y 41 estudiantes (de ambas especialidades) de la Comunidad de Madrid. Los resultados señalan que es posible identificar tres tipos de concepciones diferentes: segregadores, integradores e inclusivos, y que éstas se relacionan con la especialidad y experiencia de los participantes; se concluye la relevancia de estudiar y reformular las estrategias de formación docente de modo que potencien y fomenten la reflexión sobre la práctica.

\section{Diseño metodológico}

\section{Objetivos del estudio}

Tomando como referencia los estudios e investigaciones realizados, este trabajo pretende conocer las percepciones del alumnado del Máster Universitario en profesorado de Educación Secundaria Obligatoria y Bachillerato, Formación Profesional y Enseñanza de Idiomas acerca de la formación inicial recibida sobre 
atención a la diversidad. Así pues, la investigación intenta averiguar la finalidad así como los conocimientos adquiridos por el alumnado de este título sobre el proceso de atención a la diversidad en el aula. De este modo, y tras el análisis de resultados, es posible establecer propuestas de mejora en la formación inicial de docente de Educación Secundaria.

\section{Participantes}

La población objeto de estudio está compuesta por 108 estudiantes matriculados durante el curso académico 2012/13 en el Máster Universitario en profesorado de Educación Secundaria Obligatoria y Bachillerato, Formación Profesional y Enseñanza de Idiomas de la Universidad de Jaén. Para la selección de los sujetos se ha utilizado un muestreo probabilístico aleatorio simple, de forma que se han adscrito a la muestra todos aquellos sujetos que al azar respondieron a los cuestionarios elaborados para tal fin $(n=99)$. Para el cálculo de la muestra participante se utilizó la fórmula para poblaciones con menos de 100.000 sujetos con un nivel de confianza del $95 \%$ y un error de estimación máximo de un $4 \%$

Así pues, un $39.4 \%$ de la muestra son hombres, frente al $60.6 \%$ que está compuesto por mujeres. Además, un $63.6 \%$ de la muestra son menores de 25 años, frente al $20.2 \%$ que superan los 31 años de edad y el 16.2\% cuya edad se sitúa entre los 26 y los 30 años. El alumnado ha accedido a dicho título de posgrado tras haber cursado, en un $60.6 \%$ de los casos, una titulación de Humanidades; un $18.2 \%$ lo ha hecho en titulaciones vinculadas con Enseñanzas Técnicas, un $10.1 \%$ de Ciencias Experimentales, un $7.1 \%$ de Ciencias Sociales y Jurídicas y, finalmente, un $4.0 \%$ de Ciencias de la Salud. Dichos estudios iniciales han hecho que un 34.3\% curse este Máster para la especialidad de Lengua Extranjera, un 11.1\% para Biología y Geología, Orientación y Tecnología y Procesos Industriales, respectivamente. De igual modo, un 9.1\% cursa este título para la especialidad de Lengua y Literatura y un $6.1 \%$ para Dibujo, Imagen y Artes Plásticas y Ciencias Sociales respectivamente. Finalmente, se destaca cómo un $5.1 \%$ de la muestra cursa este título para la especialidad de Economía, Empresa y Comercio y un 3.0\% para Formación y Orientación Laboral y Matemáticas e Informática respectivamente.

Finalmente, un $48.5 \%$ de la muestra afirma haber accedido a este título para optar a un puesto de trabajo estable, frente a un $34.6 \%$ que lo hace por vocación. Además, un $6.2 \%$ se ha decidido a estudiar este Máster por influencia de un familiar vinculado al ámbito de la docencia, un $4.6 \%$ por influencia de un profesor que le ha marcado en su formación y un $1.5 \%$ que lo ha hecho por prestigio y para diversificar la docencia, respectivamente. 


\section{Instrumento}

Para la recogida de datos, se ha utilizado un cuestionario elaborado ad hoc. Dicho instrumento, compuesto de 43 ítems, consiste en una escala tipo Likert cuyas opciones de respuesta oscilan entre 1 y 4 (siendo 1 =plenamente de acuerdo, $2=$ de acuerdo; $3=$ en desacuerdo y 4 =totalmente en desacuerdo).

Construida la escala, se procedió a la validación por parte de una docena de expertos universitarios en Pedagogía de la Universidad de Jaén y Granada a los que se les pidió que valoraran y validaran la pertinencia de cada uno de los ítems del cuestionario, estableciendo las consideraciones oportunas para mejorarlo en cuanto a su contenido, ambigüedad, redacción y otros aspectos. Una vez estas sugerencias fueron incorporadas al instrumento, se procedió a la aplicación de una prueba piloto a 90 estudiantes universitarios que se encontraban cursando dicho Máster en otras universidades andaluzas. Esta prueba tenía como intención conocer el grado de comprensión de los distintos ítems por parte del alumnado y analizar la validez de constructo (análisis factorial mediante la extracción de componentes principales con rotación Varimax). Así, el índice de adecuación muestral KMO alcanza un valor de .800 y la prueba de esfericidad de Bartlett es de 3277.395 ( $p=.000)$. Estos datos hacen que se rechace la hipótesis nula de que la matriz de correlación inter-ítems es identidad y se consideran que las respuestas están sustancialmente relacionadas. El análisis de los componentes principales revela, tras la rotación Varimax, la convergencia en 5 factores que explican el $60.80 \%$ de la varianza; los ítems muestran valores apropiados, situándose entre .564 y .853 .

En cuanto a la fiabilidad del instrumento, se ha utilizado el método alpha de Cronbach obteniéndose un valor de .915. Por ello, se puede deducir que el cuestionario elaborado para tal investigación tiene una fiabilidad muy alta, ya que el coeficiente está próximo a 1, considerada la correlación perfecta. Para asegurar aún más la fiabilidad del instrumento, se aplicó el método de las dos mitades, obteniéndose puntuaciones muy apropiadas, pues en la primera parte se obtuvo un valor de .842 y para la segunda el valor obtenido fue de .906 .

\section{Procedimiento}

El plan de estudios del Máster Universitario en profesorado de Educación Secundaria Obligatoria y Bachillerato, Formación Profesional y Enseñanza Idiomas de la Universidad de Jaén, consta de un módulo genérico en el que se incluye la asignatura denominada "Procesos y contextos educativos"; es en esta materia dónde tiene cabida el estudio de contenidos propios del ámbito de la Educación Especial y la atención a la diversidad en la formación de los docentes de Educación Secundaria.

Por ello, y para la aplicación del cuestionario a la muestra, se tuvo en cuenta el que los estudiantes hubiesen cursado ya esta materia obligatoria para todas las especialidades durante el primer cuatrimestre del curso 2012/13. Asimismo, se contó con el visto bueno de los coordinadores de este Máster, los cuáles nos facilitaron el 
acceso a la muestra. Cada uno de los estudiantes recibió las explicaciones necesarias para cumplimentar el cuestionario de manera adecuada.

\section{Análisis de datos}

Para el tratamiento estadístico de los datos se ha utilizado el programa SPSS (versión 19 para Windows) al considerarlo un recurso idóneo para nuestro trabajo. De este modo, se ha llevado a cabo un análisis descriptivo de los resultados obtenidos en una de las dimensiones del cuestionario denominada: "Capacitación docente hacia la diversidad adquirida en el Máster". Además, se ha realizado un análisis de comparación de medias (ANOVA) tomando como referencia la variable sociodemográfica "especialidad del título". Se destaca como todos los análisis realizados se han calculado con un nivel de confianza del 95\%; asimismo, se ha llevado a cabo un análisis a posteriori a fin de comprobar la diferencia entre todos los pares de medias en el contexto de la muestra total; en concreto, se han asumido varianzas iguales empleando, para ello, la prueba posthoc de Tukey.

\section{Resultados}

Los resultados obtenidos permiten comprobar cómo el alumnado del Máster de Educación Secundaria considera que dicho título le ha sido de utilidad para su formación académica. Así pues, un 67.7\% del alumnado se muestra plenamente de acuerdo o de acuerdo en considerar que el Máster en Educación Secundaria le ha servido para aumentar su interés hacia la formación académica en el ámbito de la atención a la diversidad, frente al $32.3 \%$ que se muestra contrario con dicho planteamiento. Por su parte, un $66.7 \%$ del alumnado sostiene cómo este Máster lo ha aprovechado para afianzar su elección profesional hacia la docencia, mientras que el $33.4 \%$ se muestra en desacuerdo. Además, un $64.6 \%$ se muestra conforme en considerar que el hecho de cursar este título de posgrado ha repercutido en una mayor sensibilización hacia la atención a la diversidad, ofreciendo una adecuada respuesta educativa para el alumnado de Educación Secundaria Obligatoria con necesidades específicas de apoyo educativo, frente al $35.4 \%$ que se muestra reacio con dicha proposición.

No obstante, y a pesar de la utilidad del título para su formación académica, tan sólo un $43.4 \%$ se muestra conforme en considerar que, a través de este Máster, ha adquirido suficientes conocimientos sobre Educación Especial. Al cuestionar los aspectos sobre los que dicha formación versa, se destaca cómo un $66.7 \%$ se muestra de acuerdo en considerar que posee un conocimiento adecuado sobre la identificación de necesidades educativas en el alumnado; un $61.6 \%$ sobre los recursos y servicios prestados desde el sistema educativo; un 59.6\% sobre las características del alumnado con necesidades educativas; un $58.6 \%$ sobre los aspectos básicos del 
campo de la Educación Especial y, finalmente, un 57.6\% sobre la legislación relativa a dicho ámbito.

\begin{tabular}{|l|c|c|c|c|}
\hline Capacitación docente hacia la diversidad adquirida en el Máster & $\mathbf{1}$ & $\mathbf{2}$ & $\mathbf{3}$ & $\mathbf{4}$ \\
\hline $\begin{array}{l}\text { Este Máster me ha servido para aumentar mi interés hacia una mayor } \\
\text { formación en el ámbito de la atención a la diversidad }\end{array}$ & 22.2 & 45.5 & 23.2 & 9.1 \\
\hline $\begin{array}{l}\text { Este Máster me ha servido para tener una mayor sensibilización hacia } \\
\text { la atención a la diversidad }\end{array}$ & 24.2 & 40.4 & 27.3 & 8.1 \\
\hline $\begin{array}{l}\text { Este Máster me ha servido para afianzar mi elección profesional } \\
\text { hacia la docencia }\end{array}$ & 20.2 & 46.5 & 25.3 & 8.1 \\
\hline $\begin{array}{l}\text { Tras cursar el módulo genérico del Máster, poseo un conocimiento } \\
\text { adecuado sobre la identificación de necesidades educativas en el } \\
\text { alumnado }\end{array}$ & 11.1 & 55.6 & 26.3 & 7.1 \\
\hline $\begin{array}{l}\text { Tras cursar el módulo genérico del Máster, tengo un conocimiento } \\
\text { adecuado sobre los recursos y servicios prestados desde el sistema } \\
\text { educativo }\end{array}$ & 8.1 & 53.5 & 35.4 & 3.0 \\
\hline $\begin{array}{l}\text { Tras cursar el módulo genérico del Máster, poseo un conocimiento } \\
\text { adecuado sobre las características de los alumnos con necesidades } \\
\text { educativas }\end{array}$ & 10.1 & 49.5 & 36.4 & 4.0 \\
\hline $\begin{array}{l}\text { Tras cursar el módulo genérico del Máster, tengo un conocimiento } \\
\text { adecuado sobre los aspectos básicos del campo de la Educación } \\
\text { Especial }\end{array}$ & 10.1 & 48.5 & 36.4 & 5.1 \\
\hline $\begin{array}{l}\text { Tras cursar el módulo genérico del Máster, poseo un conocimiento } \\
\text { adecuado sobre legislación relativa al ámbito de la Educación } \\
\text { Especial }\end{array}$ & 5.1 & 52.5 & 36.4 & 6.1 \\
\hline $\begin{array}{l}\text { En el Máster que estoy cursando he adquirido suficientes } \\
\text { conocimientos sobre Educación Especial }\end{array}$ & 11.1 & 32.3 & 44.4 & 12.1 \\
\hline \begin{tabular}{l} 
1=Plenamente de acuerdo; 2=De acuerdo; 3=En desacuerdo y 4=Totalmente en desacuerdo \\
\hline
\end{tabular} & \\
\hline
\end{tabular}

Tabla 1. Distribución de frecuencias para la dimensión "Capacitación docente hacia la diversidad adquirida en el Máster".

Por su parte, el análisis de comparación de medias realizado (ANOVA) permite detectar la existencia de diferencias estadísticamente significativas para determinados ítems de la dimensión "Capacitación docente hacia la diversidad adquirida en el Máster" y la variable sociodemográfica "Especialidad del título". Así pues, los resultados obtenidos revelan cómo el alumnado del Máster no muestra diferencias en sus percepciones sobre la formación recibida al cuestionar si, tras cursar el módulo genérico de dicho título, posee un conocimiento adecuado sobre los aspectos básicos del campo de la Educación Especial $(F(9,89)=1.731, p=.093)$, las características del alumnado con necesidades educativas $(F(9,89)=1.656, p=.112)$, legislación relativa al ámbito de la Educación Especial $(F(8,89)=1.705, p=.100)$, recursos y servicios prestados al alumnado con necesidades educativas desde el sistema educativo $(F(8,89)=1.221, p=.292)$ e identificación de las mismas en el alumnado $(F(8,89)=1.398$, $p=.201)$. Además, tampoco se observan diferencias en las valoraciones del alumnado 
del título de posgrado al calificar como suficientes los conocimientos adquiridos sobre Educación Especial $F(8,89)=1.701, p=.101)$.

No obstante, sí que se aprecian diferencias estadísticamente significativas en las valoraciones del alumnado al valorar si dicho título de posgrado le ha servido para afianzar su elección profesional hacia la docencia $(F(9,89)=1.902, p=.003)$, tener una mayor sensibilización hacia la atención a la diversidad $(F(9,89)=2.499, p=.013)$ y aumentar su interés hacia la formación en este ámbito de estudio $F(9,89)=2.685$, $p=.008)$. Las diferencias aparecen vinculadas a aquellos ítems en los que se cuestiona la repercusión del título para la formación académica y actividad profesional del alumnado.

\begin{tabular}{|l|c|}
\hline \multicolumn{3}{|c|}{ Capacitación docente hacia la diversidad adquirida en el Máster } & ANOVA \\
\hline $\begin{array}{l}\text { Tras cursar el módulo genérico del Máster, considero que poseo un } \\
\text { conocimiento adecuado sobre: aspectos básicos del campo de la Educación } \\
\text { Especial }\end{array}$ & .093 \\
\hline $\begin{array}{l}\text { Tras cursar el módulo genérico del Máster, considero que poseo un } \\
\text { conocimiento adecuado sobre: las características de los alumnos con } \\
\text { necesidades educativas }\end{array}$ & .112 \\
\hline $\begin{array}{l}\text { Tras cursar el módulo genérico del Máster, considero que poseo un } \\
\text { conocimiento adecuado sobre: legislación sobre Educación Especial }\end{array}$ & .100 \\
\hline $\begin{array}{l}\text { Tras cursar el módulo genérico del Máster, considero que poseo un } \\
\text { conocimiento adecuado sobre: recursos y servicios prestados desde el sistema } \\
\text { educativo }\end{array}$ & .292 \\
\hline $\begin{array}{l}\text { Tras cursar el módulo genérico del Máster, considero que poseo un } \\
\text { conocimiento adecuado sobre: identificación de necesidades educativas en el } \\
\text { alumnado }\end{array}$ & .201 \\
\hline $\begin{array}{l}\text { En el Máster que estoy cursando he adquirido suficientes conocimientos sobre } \\
\text { Educación Especial }\end{array}$ & .101 \\
\hline Este Máster me ha servido para afianzar mi elección profesional hacia la docencia & $.003^{*}$ \\
\hline $\begin{array}{l}\text { Este Máster me ha servido para tener una mayor sensibilización hacia la atención } \\
\text { a la diversidad }\end{array}$ & $.013^{*}$ \\
\hline $\begin{array}{l}\text { Este Máster me ha servido para aumentar mi interés hacia una mayor formación } \\
\text { en el ámbito de la atención a la diversidad }\end{array}$ & $.008^{*}$ \\
\hline
\end{tabular}

Tabla 2. ANOVA según la especialidad del título $\left({ }^{\star} \mathrm{p}=.05\right)$

\section{Discusión y conclusiones}

Esta investigación ha permitido conocer las percepciones del alumnado del Máster de Educación Secundaria acerca de la formación inicial recibida sobre atención a la diversidad. De este modo, se intenta analizar en qué medida el futuro docente de Educación Secundaria dispone de suficientes competencias y habilidades docentes como para dar respuesta a las necesidades educativas del alumnado en dicha etapa educativa.

Los resultados obtenidos demuestran cómo el alumnado encuestado muestra una preocupación por ampliar su formación, pues no considera que el simple hecho de 
cursar este título de Posgrado lo habilite como para atender con criterios de calidad las necesidades específicas de apoyo educativo del alumnado en Educación Secundaria; además, un amplio porcentaje expone cómo este título le ha servido para aumentar su interés hacia el estudio en el ámbito de la atención a la diversidad y la Educación Especial.

Estos datos guardan relación con los aportados por Alegre (2013), cuyo estudio demuestra cómo los estudiantes del Máster de Secundaria manifiestan preocupación por mejorar su formación para responder a las peculiaridades del alumnado con necesidades de apoyo educativo. Así pues, el desarrollo de asignaturas vinculadas al ámbito de la Educación Especial y la atención a la diversidad en el aula repercute de manera directa en sus creencias y actitudes, haciéndolas más integradoras (Arranz García y Liesa, 2013; Pegalajar, Colmenero y Pérez, 2013).

No obstante, y al cuestionar la formación del alumnado del Máster sobre atención a la diversidad, los resultados obtenidos revelan cómo este título permite que el alumnado adquiera suficientes conocimientos sobre la identificación y características del alumnado con necesidades educativas, los recursos y servicios prestados desde el sistema educativo, aspectos básicos del campo de la Educación Especial y legislación educativa relativa a este ámbito de estudio. Estos datos muestran cómo la formación del título supone una convergencia de elementos teórico-prácticos, lo cuál incide de manera directa en la calidad de la formación docente para la etapa de Educación Secundaria.

Ello tiene en cuenta los resultados aportados por Colmenero (2007) quién demuestra cómo al profesorado en ejercicio le interesa cuestiones de tipo práctico y dinámico relacionadas con la atención a la diversidad. Así pues, más del $50 \%$ del alumnado considera que los conocimientos adquiridos para atender a las necesidades específicas de apoyo educativo del alumnado en las aulas de Educación Secundaria han sido suficientes. Estos datos contradicen los aportados por Sánchez y Boix (2008) quiénes exponen cómo los futuros docentes admiten tener un desconocimiento absoluto acerca del concepto de atención a la diversidad, factores y medidas de atención a la diversidad en el aula, etc. Ello repercute en la calidad del sistema educativo, pues el docente sólo puede ofrecer ayudas pedagógicas adecuadas una vez disponga de suficiente formación teórico-práctica que permita el desarrollo de un proceso de enseñanza-aprendizaje con criterios de calidad (Sykes, Bird y Kennedy, 2010; Conklin, 2012).

Además, esta investigación desprende la existencia de diferencias estadísticamente significativas en las percepciones de los futuros docentes sobre su nivel de capacitación hacia la atención a la diversidad según la especialidad por la que cursan dicho título. De igual modo, López, Echeita y Martín (2009) identifican tres tipos de concepciones diferentes hacia la atención a la diversidad (segregadoras, integradoras e inclusivas) relacionándolas con la especialidad y experiencia de los docentes. No obstante, y a pesar de considerar este factor, se debe tener en cuenta otros aspectos 
tales como las actitudes y creencias del propio docente así como la experiencia personal previa con alumnado con necesidades específicas de apoyo educativo.

A pesar de ello, la escasa muestra con la que se ha llevado a cabo dicha investigación no permite garantizar la generalización de los resultados a otras muestras. Además, el uso exclusivo del cuestionario como instrumento de recogida de datos puede generar problemas de deseabilidad social y sinceridad al cumplimentarlo. De cara a futuras investigaciones, puede resultar interesante conocer la valoración de los profesionales que imparten docencia en el Máster; analizar qué percepciones tienen hacia la diversidad así como el análisis y valoración que hacen de la formación hacia la atención a la diversidad que desarrollan en las aulas universitarias.

\section{Bibliografía}

Alegre, O. M. (2013). La formación del profesorado de Educación Secundaria en competencias vinculadas a la inclusión: el caso de la Universidad de La Laguna. En S. Castillo Arredondo (coord.). El profesor de Educación Secundaria para el siglo XXI. Fundamentos para su formación (pp. 31-44). Universidad Nacional de Educación A Distancia: Madrid.

Arranz, P.; García, E. Pascual y Liesa, M. (2013). La atención al alumnado con necesidades específicas de apoyo educativo en la formación de los futuros profesores de enseñanzas medias. En S. Castillo Arredondo (coord.). Reflexiones, Análisis y Propuestas sobre la Formación del Profesorado de Educación Secundaria (pp. 237-239). Universidad Nacional de Educación A Distancia: Madrid.

Buendía, L., Berrocal, E., Olmedo, E., Pegalajar, M., Ruiz, M. y Tomé, M. (2011). Valoración por parte del alumnado de las competencias que se pretenden conseguir con el Máster Universitario de Profesorado en Educación Secundaria Obligatoria, Bachillerato, Formación Profesional y Enseñanza de Idiomas. Bordón. Revista de Pedagogía, 63 (3), 57-74.

Calvo, A. y Susinos, T. (2010). Prácticas de investigación que escuchan la voz del alumnado: mejorar la universidad indagando la experiencia. Profesorado. Revista de Curriculum y formación del profesorado, 14 (3), 1-14.

Clotfelter, C.T; Ladd, H.F. y Vidgor, J.L. (2007). Teacher credentials and student achievement in High School: A Cross-subject analysis with student fixed effects. Disponible en: http://www.nber.org/papers/w13617.pdf?new window=1 (consultado el 15 de Febrero de 2013).

Colmenero, $M^{a}$. J. (2007). Detección de las necesidades formativas referidas al ámbito de conocimiento del profesorado de educación secundaria en procesos de atención a la diversidad. XXI Revista de Educación, 9, 207-216.

Conklin, H. (2012). Tracing learning from divergent teacher education pathways into practice in middle grades classrooms. Journal of Teacher Education, 63(3), 171-184. 
UT. Revista de Ciències de l'Educació Juny 2014. Pag. 83-96
Capacitación Docente para la Atención a la Diversidad en Futuros Docentes de Educación Secundaria

Forteza, D. (2011). Algunas claves para repensar la formación del profesorado sobre la base de la inclusión. Revista Interuniversitaria de Formación del Profesorado, 70 (25, 1), 127-144.

Gustafsson, J.E. (2003). What Do We Know About Effects of School Resources on Educational Results? Swedish Economic Policy Review, 10, 77-110.

Ley Orgánica 2/2006 de 3 de Mayo de Educación (B.O.E. 4-5-2006).

López, M., Echeita, G. y Martín, E. (2009). Concepciones sobre el proceso de inclusión educativa de alumnos con discapacidad intelectual en la educación secundaria obligatoria. C\&E: Cultura y Educación, 21 (4), 485-496.

McKinsey, C. (2007). How the World's Best Performing School Systems Come Out on Top. Disponible en: http://www.mckinsey.com/clientservice/socialsector/resources/pdf/Worlds_School_Sy stems Final.pdf (consultado el 7 de Mayo de 2014).

Orden ECI/3858/2007 de 27 de diciembre por la que se establecen los requisitos para la verificación de los títulos universitarios oficiales que habiliten para el ejercicio de las profesiones de Profesor de Educación Secundaria Obligatoria y Bachillerato, Formación Profesional y Enseñanzas de Idiomas (B.O.E. 29-12-2007).

Pegalajar, M.C.; Colmenero, Ma.J. y Pérez, E. (2013). Necesidades formativas del profesorado de educación secundaria sobre atención a la diversidad. En S. Castillo (coord.). Reflexiones, Análisis y Propuestas sobre la Formación del Profesorado de Educación Secundaria (pp. 118-120). Universidad Nacional de Educación A Distancia: Madrid.

Pérez, A.I. (2010). Aprender a educar. Nuevos desafíos para la formación de docentes. Revista Interuniversitaria de Formación del Profesorado, 68 (24,2), 37-60.

Pestano, M.A. y Alegre, O.M. (2013). La formación del profesorado de Educación Secundaria en actitudes hacia la inclusión e interculturalidad de la universidad de La Laguna. En S. Castillo Arredondo (coord.). Reflexiones, Análisis y Propuestas sobre la Formación del Profesorado de Educación Secundaria (pp. 164-167). Universidad Nacional de Educación A Distancia: Madrid.

Rocard, M., Csermely, P., Jorde, D., Lenzen, D., Walwerg-Henriksson, H, y Hemmo, V. (2007). Science Education Now: A Renewed Pedagogy for the Future of Europe. European Commission. Community Research.

Sánchez, A. (2007). Investigación sobre la formación inicial del profesorado de educación secundaria para la atención educativa a los estudiantes con necesidades educativas. Revista Interuniversitaria de formación del profesorado, 21 (2), 149-181.

Sánchez, A. y Boix, J.L. (2008). Los futuros profesores de educación secundaria: inicio de su profesionalización y construcción de su identidad docente. Revista Electrónica Interuniversitaria de Formación del profesorado, 11 (2), 31-45. 
Santos, M.A. (2010). La formación del profesorado en las instituciones que aprenden. Revista Interuniversitaria de Formación del Profesorado, 68 (24,2), 175-200.

Schneider, M. y Stern, E. (2010). The cognitive perspective on learning: ten cornerstone findings. En Organisation for Economic Co-Operation and Development (OECD) (Ed.). The nature of learning: Using research to inspire practice (pp. 69-90). Paris: OECD.

Sykes, G., Bird, T. y Kennedy, M. (2010). Teacher education: Its problems and some prospects. Journal of Teacher Education, 61, 464-476.

Vigo, B., Soriano, J. y Julve, C. (2010). Preparando profesores para la atención a la diversidad: potencialidades y limitaciones de un proyecto de innovación y mejora interdisciplinar. Revista Interuniversitaria de Formación del Profesorado, 69 (24,3), 147 165.

Vilchez, A. y Gil, D. (2007). La necesaria renovación de la formación del profesorado para una educación científica de calidad. Tecné, Episteme y Didacxis, 22, 67-85.

\section{Nota biográfica}

$\mathbf{M}^{\mathbf{a}}$ Jesús Colmenero Ruiz. Doctora en Pedagogía, Licenciada en Filosofía y Letras y Ciencias de la Educación. Profesora del Departamento de Pedagogía de la Universidad de Jaén. Las líneas de investigación están centradas en la formación del profesorado, procesos de atención a la diversidad e inclusión escolar. Secretaria de la revista de Educación Inclusiva.

mjruiz@ujaen.es

Universidad de Jaén. Facultad de Humanidades y Ciencias de la Educación. Departamento de Pedagogía. Edif C5. Despacho 237. Campus Las Lagunillas s/n. 23071 Jaén

$\mathbf{M}^{\mathbf{a}}$ del Carmen Pegalajar Palomino. Doctora en Educación, Licenciada en Psicopedagogía y Diplomada en Maestro, especialidad en Educación Primaria. Máster en Educación Especial, profesora del Departamento de Educación de la Universidad Católica de Murcia. Sus líneas de investigación están centradas en la atención a la diversidad y la formación del profesorado en el ámbito de la Educación Especial.

mdcpegalajar@ucam.edu

Universidad Católica de Murcia. Departamento de Magisterio. Campus "Los Jerónimos", s/n. 30107 Guadalupe (Murcia). 